\title{
CPTAC Cervical Squamous Cell Carcinoma and Endocervical Adenocarcinoma Baseline Form
}

National Cancer Institute

\section{Source}

National Cancer Institute. CPTAC Cervical Squamous Cell Carcinoma and Endocervical

Adenocarcinoma Baseline Form. NCI Thesaurus. Code C157526.

Terminology used in support of the data collection efforts of the Clinical Proteomic

Tumor Analysis Consortium (CPTAC) with the focus on Cervical Squamous Cell

Carcinoma and Endocervical Adenocarcinoma Baseline Form data. 